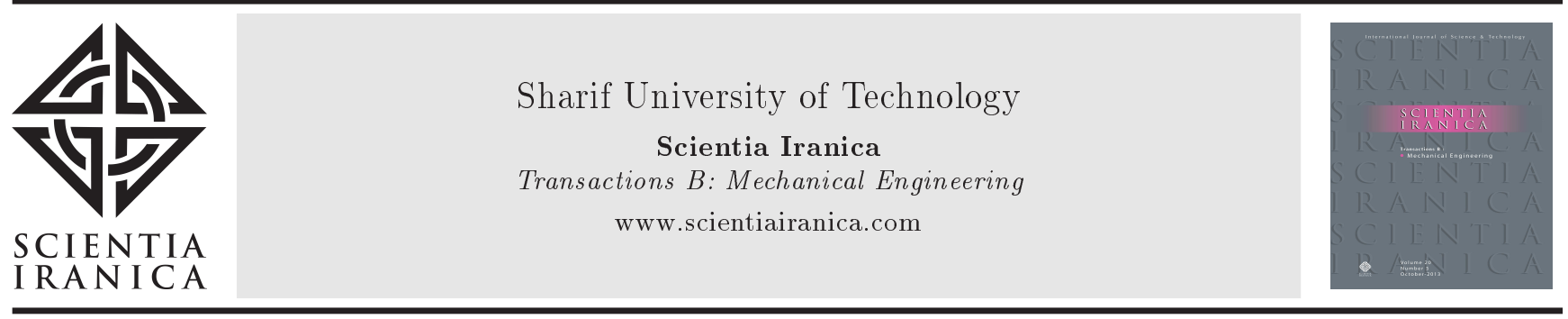

\title{
Effects of geometrical parameters on the performance of a cold gas micronozzle
}

\author{
M.M. Heydari* \\ Propulsion Group, Aerospace Complex, Malek-Ashtar University of Technology, Tehran, Iran.
}

Received 3 May 2014; received in revised form 18 June 2015; accepted 10 August 2015

\section{KEYWORDS}

Micronozzle;

Gas flow;

Slip conditions;

Numerical simulation.

\begin{abstract}
The area of the exit and throat region of a nozzle play a crucial role in its design. This paper is a report of numerical simulations carried out to investigate the influence of these parameters on the performance factors of an axisymmetric cold gas nozzle of micron-size throat diameter. It was assumed that the deviations of the flow behavior from that of a continuum flow can be taken care of by applying the first-order slip boundary conditions at the wall. The solution methodology includes a finite-volume-based numerical procedure based on structured quadrilateral grids. A parametric study reveals that to reach the highest values of the thrust and specific impulse, one should choose a nozzle with the highest possible throat diameter. However, by increasing the outlet diameter, the thrust initially reaches a maximum and then decreases. In these conditions, the specific impulse is always a decreasing function of the outlet diameter of the micronozzle. It is also observed that the mass flow rate is an increasing function of both the throat and outlet diameters. In addition, the comparison of the results with and without slip velocity shows that the amounts of the mass flow rate, thrust force, and specific impulse are higher when the rarefaction effects are taken into account. Nevertheless, no fundamental difference is observed in flow physics with and without slip velocity.

(C) 2016 Sharif University of Technology. All rights reserved.
\end{abstract}

\section{Introduction}

During the last two decades, the improvements in microfabrication techniques have led to the development of an increasing number of microfluidic technologies. Many microfluidic systems are potential application areas for gaseous microflows, such as miniaturized heat exchangers for cooling of integrated circuits, portable gas chromatography systems for the detection of airborne pollutants, microreactors for generating small quantities of dangerous or expensive chemicals, and novel high-throughput gas flow cytometers [1].

Because of significant rarefaction effects, the flow physics of gas flow at microscale can be quite different from those at macroscale. The features of

*. Tel.: +982166065030

E-mail address: mmheydary@gmail.com this difference, which have been justified by a lot of experiments [2-7], include discontinuities of velocity and temperature on the boundary, non-Newtonian components of stress tensor, non-Fourier heat flux, and formation of Knudsen boundary layer [8]. Based on the degree of rarefaction, some of the aforementioned features are present. The degree of rarefaction is measured by the Knudsen number, defined as $\mathrm{Kn}=$ $\lambda / L$, with $L$ denoting the characteristic length scale of the device and $\lambda$ being the mean free path of the gas molecules. The mean free path is given by $\lambda=$ $\mu \sqrt{\pi / 2 R T \rho^{2}}$, where $\mu$ is the dynamic viscosity, $R$ is the gas constant, and $T$ and $\rho$ are temperature and density of the gas, respectively [9].

Based on a classification given by Beskok and Karniadakis [10], gas flow can be categorized into four regimes according to its Knudsen number. In the slip flow regime, corresponding to $10^{-3} \leq \mathrm{Kn} \leq 0.1$, which 
is the subject of the present investigation, deviations from the state of continuum are relatively small and the Navier-Stokes equations are still valid, except at the region next to the boundary which is known as Knudsen boundary layer. The Knudsen boundary layer is significant only up to distances of the order of one mean free path from the wall [11]. Hence, besides the velocity and temperature discontinuities at the wall, its effects are negligible in slip flow regime and the Navier-Stokes equations may be applied to the whole domain. The velocity and temperature discontinuities are incorporated into the solution as boundary conditions [12-14].

With the advent of micro- and nanosatellites in recent years, the need for the development of miniaturized propulsion devices based on Micro-ElectroMechanical Systems (MEMS) has increased [15-18]. A MEMS-based propulsion system might consist of an array of tiny rocket thrusters on a silicon chip with electronic circuitry that controls firing. Currently, various micropropulsion concepts are being considered, such as cold gas, catalytic decomposition, vaporizing liquid, and mono- and bipropellant thrusters [19]. Despite the fact that numerous research works have been reported in the literature on different aspects of gas flow in microchannels [20-38], the studies that address gas flow in microthrusters are still rare. One of the first attempts in this respect was made by Rossi et al. [39]. They presented a new model for predicting the processes inside the thruster based on the computation of the unsteady gas flow inside the microthruster. They, however, assumed the validity of the no-slip conditions at the wall. This study was followed by several research works by Alexeenko et al. [19,40,41]. They made use of both continuum and Direct Simulation Monte Carlo (DSMC) methods to explore the performance factor of different micronozzles under both steady and transient conditions. The same methods were exploited by Liu et al. [42] in order to study the flow through a two-dimensional micronozzle by applying slip boundary conditions. It was found that within the Knudsen number range under consideration, both methods worked to predict the flow characteristics inside micronozzles. A DSMC-based study, performed by Chong [43], revealed that the statistical method can deal well with the near-sonic flows in MEMSbased propulsion systems. More recently, a hybrid CFD/DSMC methodology was utilized by Torre et al. [44] to study the flow within an axisymmetric micronozzle. A CFD solver was applied upstream from a properly chosen cross-sectional (perpendicular to the nozzle axis) interface, and a Direct Simulation Monte Carlo solver was applied downstream from that interface. There have also been some very recent works reported by Watvisave et al. [45] and Sebastião and Santos [46]. These works deal with the effects of wall accommodation and surface discontinuity on the performance factors of two-dimensional micronozzles, respectively.

The geometrical configurations of a micronozzle, such as its throat and exit areas, play crucial roles in nozzle design [47]. However, no study has been undertaken that addresses the influence of these parameters on the performance of a micronozzle. In this paper, a parametric study is conducted in order to find out the effects of throat and outlet diameters on the performance factors of a micronozzle utilizing the nozzle proposed by Liu et al. [42]. Since most of the relevant works in the literature deal with two-dimensional micronozzles, an axisymmetric version of the previously mentioned nozzle is considered here. As Liu et al. [42] reported the validity of the continuum analysis for their proposed nozzle, a finite volume based numerical model is used here for handling the problem by considering slip boundary conditions at the wall. The formulation of the problem is outlined in the next section.

\section{Problem formulation}

Consideration is given to compressible gas flow in a convergent-divergent nozzle of micron-size throat diameter, outlined in Figure 1. Note that this figure is taken from the work of Liu et al. [42] which actually represented a two-dimensional nozzle; nevertheless, we consider it to be an axisymmetric micronozzle and a two-dimensional domain is only considered for validation of the results in Section 4.1. The working gas, assumed to be nitrogen, possesses constant thermophysical properties and follows ideal gas behavior. The flow is considered to be steady and laminar. It is assumed that the nozzle wall is under adiabatic thermal conditions. Owing to axial symmetry of the geometry, an axisymmetric flow solver is used in this analysis.

\subsection{Mathematical modeling}

The compressible form of the Navier-Stokes equations along with the conservation of mass and energy equations is applied to model the flow. The conservation of

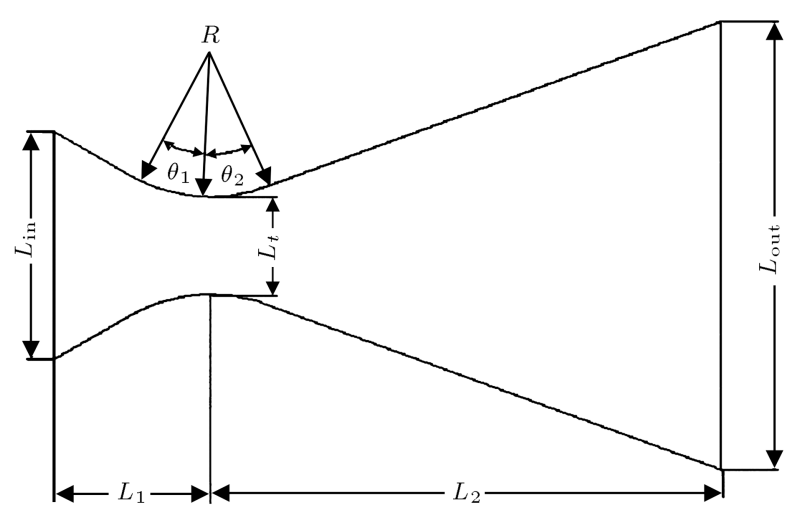

Figure 1. Micronozzle configuration [42]. 
mass at steady-state conditions is:

$$
\nabla \cdot(\overrightarrow{\exists v})=0
$$

where $v$ is the velocity vector. The conservation of linear momentum in conservative form, which is suitable for finite volume methods, is also written as:

$$
\nabla \cdot(p v v)=-\nabla p+\nabla \cdot \tau+\rho f .
$$

In Eq. (2), $p$ is the static pressure, $\tau$ denotes the stress tensor, and $f$ is the body force per unit mass which is zero in this study. For a Newtonian fluid, the relationship between $\tau$ and the velocity components is:

$$
\tau=\mu\left(\nabla v+\nabla v^{T}-\frac{2}{3} \nabla \cdot v I\right) .
$$

The compressibility effects are encountered in gas flows at high velocity and/or for cases in which there are large pressure variations. When the flow velocity approaches or exceeds the speed of sound of the gas or when the pressure change in the system $(\nabla p / p)$ is large, the variation of gas density with pressure has a significant impact on the flow velocity, pressure, and temperature. The energy equation, written in Eq. (4), incorporates the coupling between the flow velocity and static temperature, and is solved simultaneously with the continuity and momentum equations:

$$
\nabla \cdot[v(\rho E+p)]=\nabla \cdot(k \nabla T+\tau \cdot v)+q_{H},
$$

where $k$ is thermal conductivity and the first two terms on the right-hand side of Eq. (4) represent energy transfer due to conduction and viscous dissipation, respectively. Also, $q_{H}$ is the rate of volumetric heat generation due to the sources other than viscous heating, which are assumed absent in the present study. In addition, $E$ is the internal energy per unit mass and is defined as:

$$
E=h-\frac{p}{\rho}+\frac{v^{2}}{2},
$$

where $h$ is the sensible enthalpy for ideal gasses. Finally, the ideal gas law is written in the form:

$$
\rho=\frac{M_{w} p}{R T} .
$$

Here, $M_{w}$ is the molecular weight. The temperature will be computed from the energy equation.

\subsection{Boundary conditions}

As stated before, because of rarefaction effects, the velocity and temperature of the gas at the solid surface are different from the corresponding values of the wall. The velocity of the gas at the wall is known as the slip velocity and the difference between the wall temperature and the gas temperature at the wall is known as the temperature jump. The following slip relation is used to model the slip velocity at the nozzle wall [10]:

$$
v_{\text {slip }}=\frac{2-F_{m}}{F_{m}}\left(\lambda \frac{\partial v_{s}}{\partial n}+\frac{\lambda^{2}}{2} \frac{\partial^{2} v_{s}}{\partial n^{2}}+\ldots\right),
$$

where $n$ is the normal direction of the wall, and $v_{s}$ is the velocity vector component in the tangential direction $s$. Moreover, $F_{m}$ is the fraction of the molecules reflected from the wall with an average tangential velocity corresponding to that of the wall and it is called the tangential momentum accommodation coefficient. Also, $\left(1-F_{m}\right)$ is the fraction of the molecules reflected from the wall conserving their average incoming tangential velocity [48]. In this study, it is assumed that $F_{m}=1$, which means that all the momentum of the gas molecules is transferred to the wall. In this case, there is a diffuse reflection at the wall. If Eq. (7) is truncated to include only up to the firstorder terms in $\lambda$ and the curvature effects are taken into account, we recover Maxwell's first-order slip boundary condition [9] which is imposed on the wall boundaries as the velocity boundary condition:

$$
v_{\text {slip }}=\frac{2-F_{m}}{F_{m}} \lambda\left(\frac{\partial v_{s}}{\partial n}+\frac{\partial v_{n}}{\partial s}\right)
$$

It is noteworthy that because of the assumed adiabatic thermal boundary conditions, there will be no temperature jump at the wall and, hence, we do not speak about the temperature jump expression.

The problem will be well posed if the full information on the ingoing and outgoing characteristics can be recovered from the imposed combinations of conservative variables. At a subsonic inlet, the choice $(u, p)$ as boundary conditions is not well posed, but any other combination involving $\rho$ as a physical condition will result in a well posed problem. In this study, the pressure and total temperature at the inlet are specified. The inlet density can be obtained from the inlet pressure $\left(p_{\text {in }}\right)$ and temperature $\left(T_{\mathrm{in}}\right)$ using Eq. (6). The pressure at the nozzle exit is used for calculations as long as the flow is subsonic. For a supersonic flow at the outlet, there is no need to impose any boundary conditions and the exit values of flow parameters are obtained by the extrapolation of internal domain information $[49,50]$.

\subsection{Micronozzle performance}

One of the most important performance parameters when dealing with propulsion systems is the thrust force. For a steadily operating propulsion system moving through a homogeneous atmosphere, the total 
thrust is equal to:

$$
F_{t}=\int_{A_{\mathrm{out}}}\left(\rho_{e} v_{e}^{2}+p_{e}-p_{0}\right) d A,
$$

where $\rho_{e}, v_{e}$, and $p_{e}$ are the outlet values of density, velocity normal to the boundary, and pressure, respectively. The first term is the momentum thrust represented by the product of the propellant mass flow rate and its exhaust velocity relative to the vehicle. The second term represents the pressure thrust consisting of the product of the cross-sectional area at the nozzle exit.

The specific impulse $I_{s p}$ is the total impulse per unit weight of propellant. It is an important figure of merit for the performance of a propulsion system [51]. The specific impulse is computed from the relation:

$$
I_{s p}=\frac{F_{t}}{\dot{m} g},
$$

with $\dot{m}$ standing for the mass flow rate and $g$ representing the standard acceleration of gravity $\left(9.81 \mathrm{~m} / \mathrm{s}^{2}\right)$.

\section{Numerical procedure}

The continuity, momentum, and energy equations subject to the boundary conditions provided in Section 2.2 were numerically solved to simulate the flow within the micronozzle. These equations were discretized by means of the finite volume method and the coupling between the momentum and continuity equations was handled through a semi-implicit pressure based method like SIMPLE [52] which is very usual and robust in the single-phase flow simulation. The convection terms in all equations were discretized using the second-order upwind scheme. The solution was considered to be converged when the scaled residual became less than $10^{-6}$ for the continuity and momentum equations and lower than $10^{-8}$ for the energy equation.

After discretization, the conservation equation for a general variable $\phi$ at a cell $P$ can be written as [52]:

$$
a_{P} \phi_{P}=\sum_{n b} a_{n b} \phi_{n b}+b
$$

wherein $a_{P}$ is the center coefficient and $a_{n b}$ is the coefficient for neighboring cells. The residual is computed as the sum of imbalance in Eq. (11) over all the computational cells. The scaled residual is defined as:

$$
R^{\phi}=\frac{\sum_{\text {cells }}\left|\sum_{n b} a_{n b} \phi_{n b}+b-a_{P} \phi_{P}\right|}{\sum_{\text {cells }}\left|a_{P} \phi_{P}\right|} .
$$

For the continuity equation, the un-scaled residual is defined as:

$$
R^{c}=\sum_{\text {cells } P} \mid \text { rate of mass creation in cell } P \mid .
$$

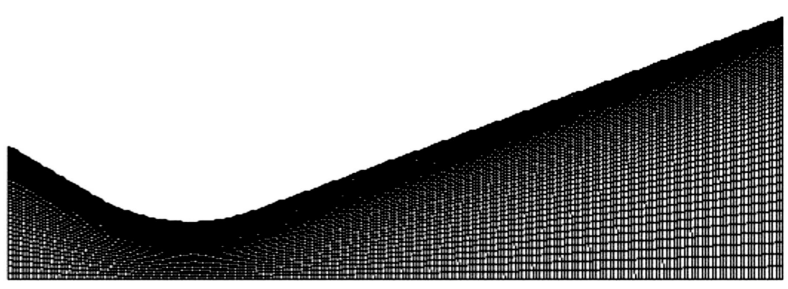

Figure 2. Computational grid (structured quadrilateral cells).

The scaled residual for the continuity equation is:

$$
\frac{R_{\text {iteration } N}^{c}}{R_{\text {iteration } 5}^{c}} \text {. }
$$

The computational grid, shown in Figure 2, consists of structured quadrilateral grid with 80 boundary layer grid cells in the radial direction and about 200 grid cells in the axial direction. Grid independency was checked by doubling the number of cells in each direction and negligible difference was observed between the results.

\section{Results and discussion}

\subsection{Two-dimensional micronozzle}

To validate our simulation, the Mach number profile at the exit plane of the nozzle assuming a two-dimensional nozzle is compared with the results of Liu et al. [42]. The comparison for the no-slip and slip conditions at the wall is shown in Figures 3 and 4, respectively. The good agreement between the results in both cases indicates the validity of the present results. The geometrical parameters of the nozzle in this section are provided in Table 1. The temperature of nitrogen gas at the inlet is $300 \mathrm{~K}$ and the inlet total (stagnation) and outlet static pressures are considered $10000 \mathrm{~Pa}$ and $10 \mathrm{~Pa}$, respectively. The value of the specified static pressure was used only when the flow was subsonic. If the flow became locally supersonic, the specified

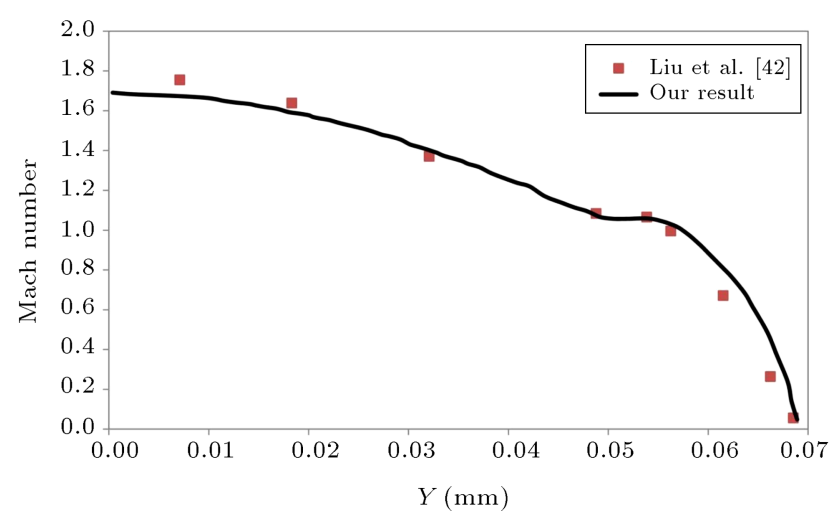

Figure 3. Comparison between the Mach number values at the exit plane of the micronozzle obtained in the present study assuming the validity of no-slip conditions and those reported by Liu et al. [42]. Here, $Y$ is the distance from the centerline of the nozzle. 


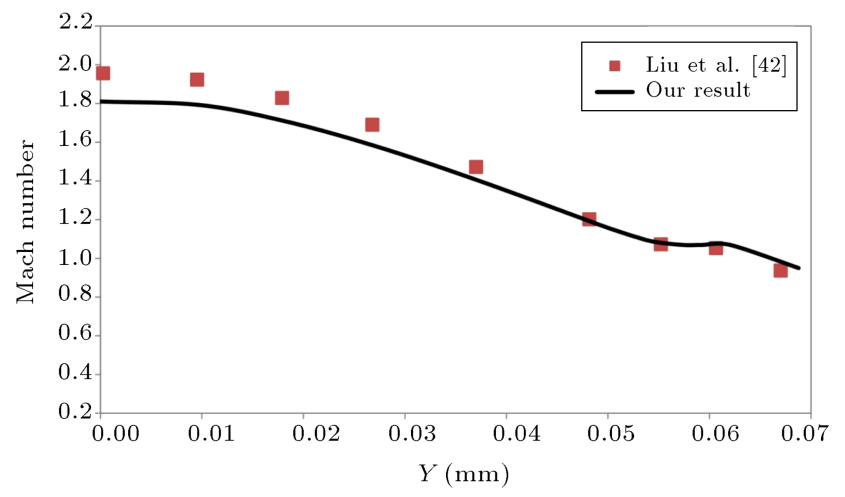

Figure 4. Comparison between the Mach number values at the exit plane of the micronozzle obtained in the present study considering the slip condition effects and those reported by Liu et al. [42]. Here, $Y$ is the distance from the centerline of the nozzle.

Table 1. Geometrical parameters of microthruster for the base case.

\begin{tabular}{cc}
\hline$L_{\text {in }}$ & $70 \mu \mathrm{m}$ \\
$L_{\text {out }}$ & $138 \mu \mathrm{m}$ \\
$L_{t}$ & $30 \mu \mathrm{m}$ \\
$R$ & $50 \mu \mathrm{m}$ \\
$L_{1}$ & $48 \mu \mathrm{m}$ \\
$L_{2}$ & $157 \mu \mathrm{m}$ \\
$\theta_{1}$ & $30^{\circ}$ \\
$\theta_{2}$ & $20^{\circ}$ \\
\hline
\end{tabular}

pressure was no longer used; the pressure and all other flow quantities were extrapolated from the flow in the interior.

It can be seen in Figures 3 and 4 that when the no-slip boundary condition is imposed at the wall, the Mach number gradient is higher near the wall, whereas the profile has smaller gradients near the center of nozzle. The flow is also subsonic near the wall and it becomes supersonic around the nozzle centerline. As can be observed in Figure 4, the Mach number profile is flatter when slip occurs at the wall. The flow is also supersonic all the way through the exit plane.

The Mach number profile at the centerline is shown in Figure 5. Despite the quasi one-dimensional inviscid flow where the sonic point is at the throat, the flow at the centerline becomes slightly supersonic after the throat. The reason behind this difference is the viscous effects; the pertinent mechanism will be discussed in the following. It is clear that in the presence of viscosity, a viscous boundary layer is formed at the wall. The flow outside this layer can be assumed inviscid. With the viscous layer, the boundary of the inviscid flow is modified. Because of high velocity flow, the distribution of the viscous layer thickness is not symmetric with respect to the throat and is therefore thicker after the throat. The boundary of the viscous

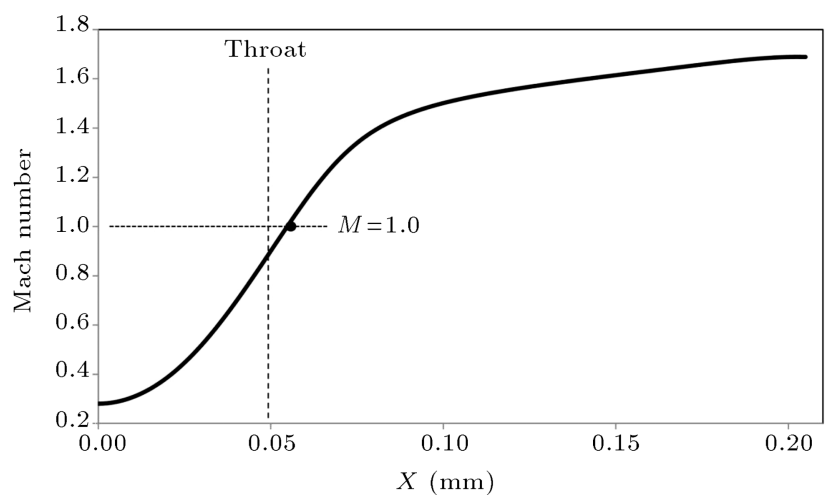

Figure 5. Mach number profile at the centerline of two-dimensional micronozzle.

layer, therefore, can be assumed to form a virtual nozzle with its throat located a little at the right of the original nozzle. This is why the flow at the centerline becomes slightly supersonic after the throat.

\subsection{Parametric study of axisymmetric micronozzle}

In this section, the inlet gas temperature and static pressure are $300 \mathrm{~K}$ and $10000 \mathrm{~Pa}$, respectively. The outlet pressure is also $1000 \mathrm{~Pa}$. We begin the presentation of the results for the axisymmetric nozzle with giving the axial velocity contours for both the slip and no-slip conditions in Figure 6. As it can be seen, in the presence of rarefaction, the axial velocity contours intersect the nozzle wall, reflecting a nonuniform velocity at the wall. This is reasonable as the difference in the shear rates at the wall causes different slip velocities throughout the wall according to Eq. (8).

Figure 7 shows the static pressure distribution inside the micronozzle. There is a gradual variation in the static pressure from the inlet to the throat and after that, the static pressure drastically decreases up to the

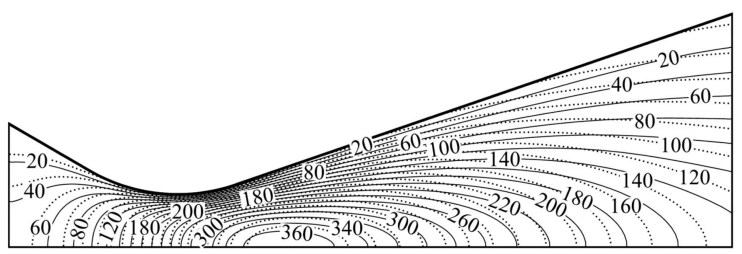

Figure 6. Axial velocity (in $\mathrm{m} / \mathrm{s}$ ) contours for axisymmetric micronozzle. Dotted lines: slip conditions; solid lines: no-slip conditions.

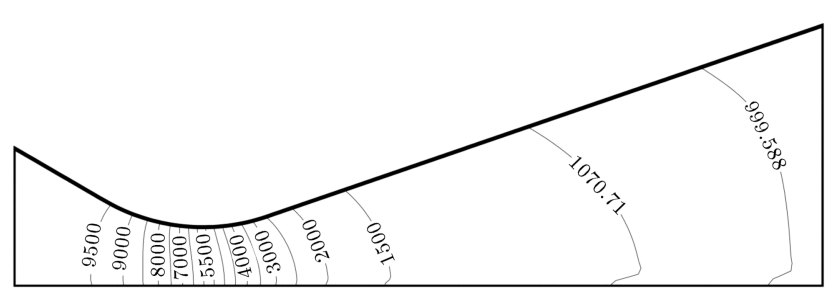

Figure 7. Pressure (in $\mathrm{Pa}$ ) distribution for axisymmetric micronozzle. 


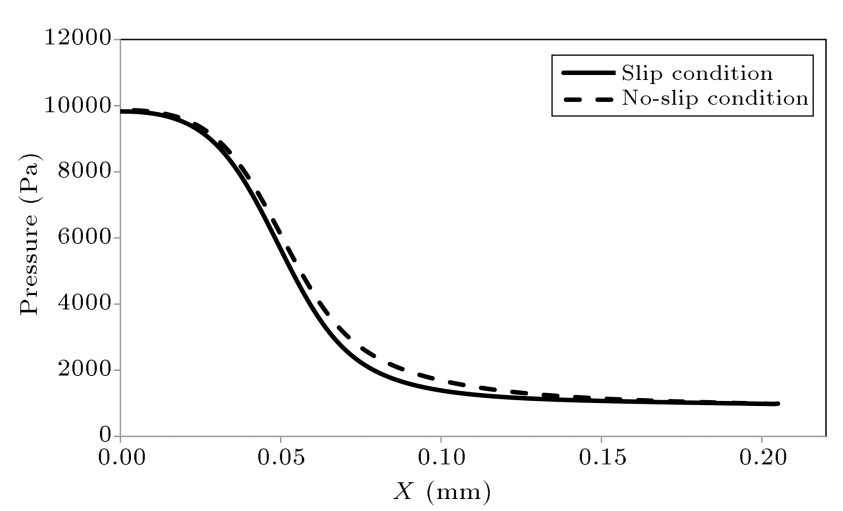

Figure 8. Pressure distribution at the centerline of axisymmetric micronozzle.

exit of micronozzle. The constant pressure line is nearly vertical, which means the pressure gradient is small in the radial direction. The axial distribution of the static pressure at the centerline is also given in Figure 8. The relevant results for the no-slip conditions are also depicted in this graph for comparison. Whereas the pressure is the same for both cases at the two nozzle ends, due to the fixed boundary conditions, it is a little higher at the other points for the no-slip conditions, especially at the vicinity of the throat. This may be attributed to the higher axial gradients of the velocity near the throat in the presence of rarefaction.

\subsubsection{Effect of throat diameter on micronozzle performance}

In this section, the effect of the throat diameter on the thrust and specific impulse of micronozzle is discussed. The throat diameter and convergence half angle are specified in Table 2. The outlet diameter and nozzle length are same as the values provided in Table 1. The values of the mass flow rate, thrust, and specific impulse corresponding to different throat diameters are also provided in Table 2. The results are also shown in Figures 9 and 10. Note that although throughout this section the no-slip condition results will also be presented for comparison, the tabular data is only given for rarefied conditions. Figures 9 and 10 indicate that both thrust force and mass flow rate increase noticeably with the throat diameter, but the thrust

Table 2. Effects of throat diameter on microthruster performance. Note that $I_{s p}$ is given by Eq. (10) with $g=9.81 \mathrm{~m} / \mathrm{s}^{2}$.

\begin{tabular}{cccccc}
\hline $\begin{array}{c}\text { Case } \\
\text { no. }\end{array}$ & $\begin{array}{c}\boldsymbol{L}_{\boldsymbol{t}} \\
(\mu \mathbf{m})\end{array}$ & $\boldsymbol{\theta}_{\mathbf{1}}$ & $\begin{array}{c}\boldsymbol{F}_{\boldsymbol{t}} \\
\left(\boldsymbol{N} \times \mathbf{1 0}^{\mathbf{6}}\right)\end{array}$ & $\begin{array}{c}\dot{\boldsymbol{m}} \\
\left(\mathbf{k g} / \mathbf{s} \times \mathbf{1 0}^{\mathbf{8}}\right)\end{array}$ & $\begin{array}{c}\boldsymbol{I}_{\boldsymbol{s p}} \\
(\mathbf{s})\end{array}$ \\
\hline 1 & 16.32 & 44 & 0.05 & 0.25 & 1.97 \\
2 & 25.86 & 34 & 1.97 & 0.75 & 26.65 \\
3 & 33.32 & 27 & 4.06 & 1.37 & 30.1 \\
4 & 57.48 & 8 & 13.89 & 4.63 & 30.59 \\
5 & 70 & 0 & 21.99 & 6.38 & 35.16 \\
\hline
\end{tabular}

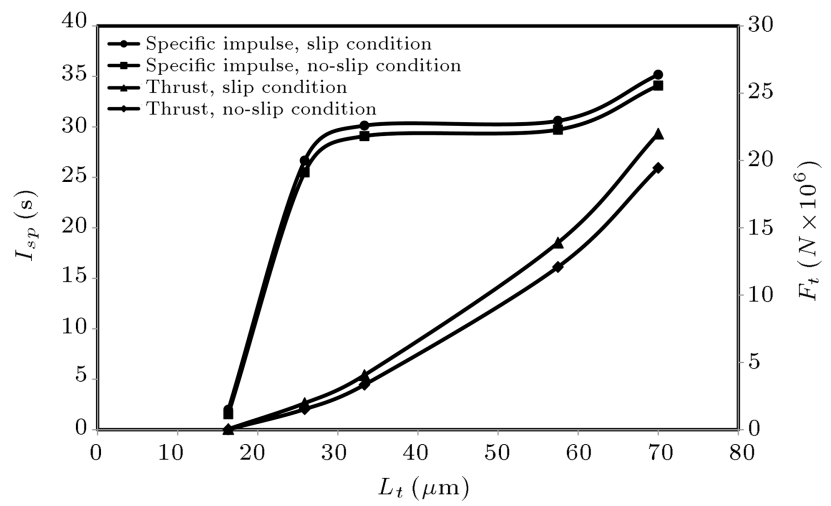

Figure 9. Effect of throat diameter on specific impulse and thrust force. Note that $I_{s p}$ is given by Eq. (10) with $g=9.81 \mathrm{~m} / \mathrm{s}^{2}$.

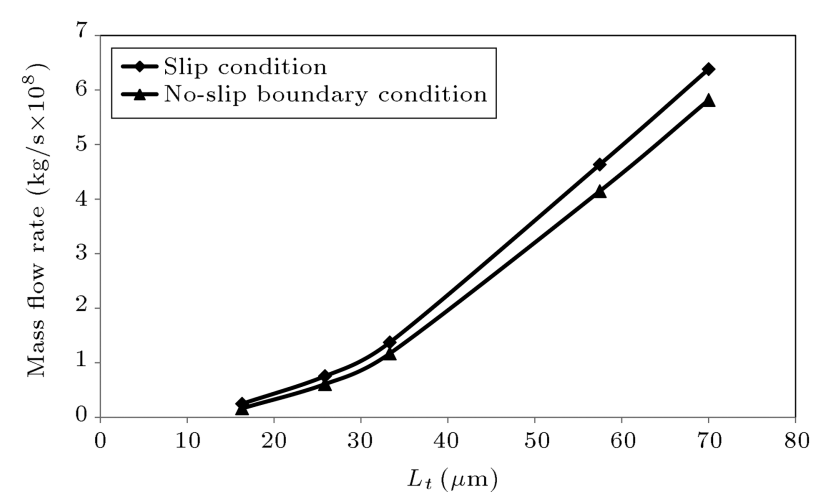

Figure 10. Effect of throat diameter on mass flow rate.

force increment is more significant so that the specific impulse also increases with the throat diameter.

To describe the reason of the increase in thrust force with the throat diameter, the Mach number distribution in the form of iso-contours for Cases 1 and 5 with throat diameters of 16 and $70 \mu \mathrm{m}$ is shown in Figure 11(a) and (b), respectively. As observed, the nozzle shown in Figure 11(a) has a larger divergence half angle and the flow nearly becomes separated, so the Mach number is very small (nearly zero) in a noticeable portion of nozzle which results in a reduction in the thrust force.

It can also be deduced from Figures 9 and 10 that the values of all the considered parameters are higher in the presence of slip conditions. The slip conditions tend to decrease the fluid-wall interaction, thereby decreasing the drag force against flow. This, for a given driving force, which is assumed here by fixing the inlet and outlet pressures, gives rise to higher velocities and, ultimately, higher amounts of the mass flow rate and the thrust force. Moreover, since the slip effects are more pronounced than the mass flow rate for the thrust force (note that this parameter contains the square of velocity), the specific impulse increases as well.

The Mach number profile at the nozzle axis is 


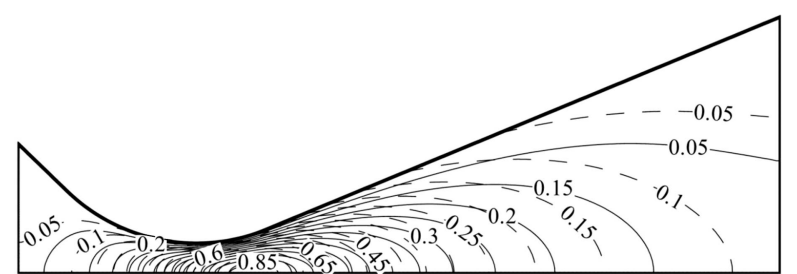

(a)

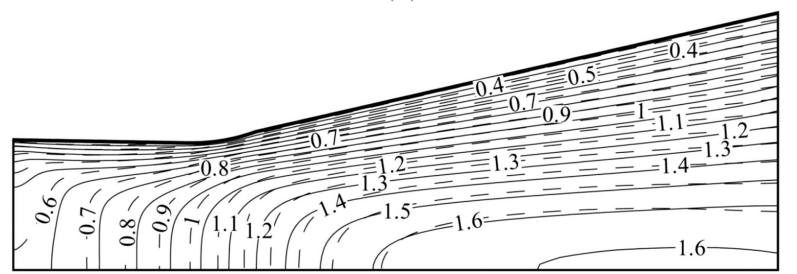

(b)

Figure 11. Mach number contours for micronozzle with throat diameter of (a) $16 \mu \mathrm{m}$, and (b) $70 \mu \mathrm{m}$. Dotted lines: slip conditions; solid lines: no-slip conditions.

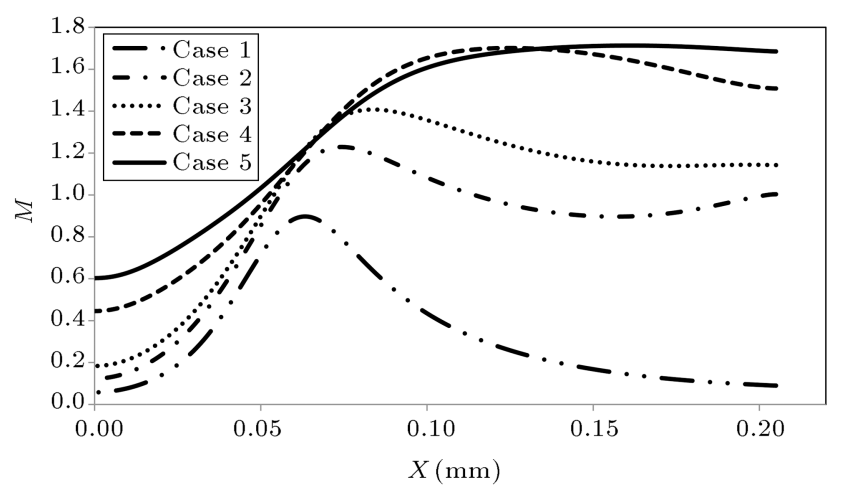

Figure 12. Mach number profile at the micronozzle axis for different throat diameters.

shown in Figure 12 for five different cases listed in Table 2. As the throat diameter increases, the Mach number at the nozzle outlet also increases. When the throat diameter is $16 \mu \mathrm{m}$, the Mach number increases in the converging part of nozzle, and then it starts to decrease again in the diverging part. The same is true to some extent for Cases 2-4, despite the fact that the flow becomes supersonic near the throat. This seemingly unexpected trend, which contradicts the predictions of

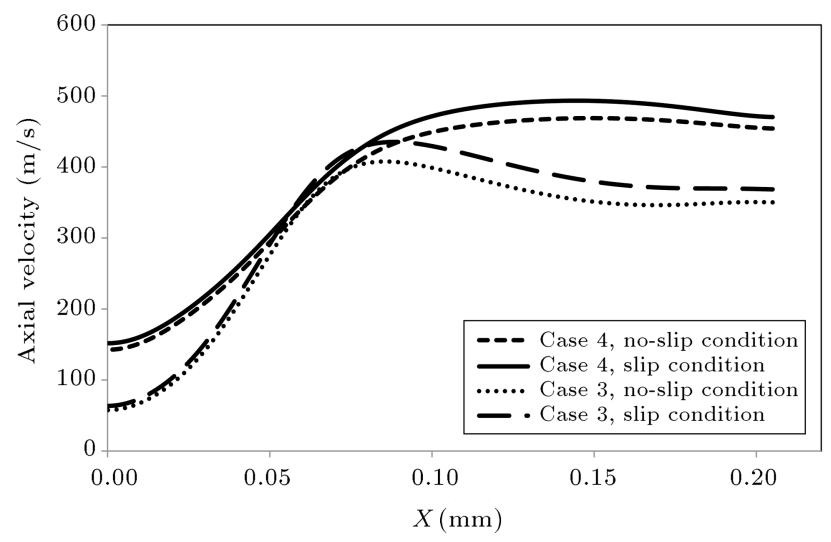

Figure 13. Axial velocity profile at the micronozzle axis for different throat diameters.

inviscid flow theory, is a consequence of viscous effects. When the viscous friction is taken into account, the expansion process may be overwhelmed, leading to a decrease in the Mach number [53]. The predictions of the inviscid flow theory may be valid to some extent even in the presence of the viscous friction, provided that the flow inertia is high enough to overcome the viscous effects, as it is the case for the micronozzle with the throat diameter of $70 \mu \mathrm{m}$, showing a totally increasing trend for the Mach number. However, the isentropic computation for choked flow (sonic velocity at the throat) shows that the outlet Mach number should be 4.51, 3.31, and 2.91 for Cases 3, 4, and 5 , respectively. Because of the viscous effects, which are significant at the microscale, the obtained Mach numbers are smaller than the isentropic values.

Figure 13, depicting the axial velocity at the centerline, shows that the trends of the results are nearly the same for both the slip and no-slip flows, revealing that they share the same flow physics. This is not much surprising as in both cases, the gas is assumed to be a continuum medium and the only difference is the different boundary conditions.

The effect of the throat diameter on the nozzle velocity and kinetic energy efficiencies is also shown in Table 3. The velocity efficiency (actual average exit velocity component parallel to the nozzle centerline

Table 3. Effects of throat diameter on microthruster efficiency. The definitions of the used velocity efficiency and kinetic energy efficiency are $\int_{A_{\text {out }}}\left(v_{\text {axial }}\right)_{\text {actual }} d A / \int_{A_{\text {out }}}\left(v_{\text {axial }}\right)_{\text {inviscid }} d A$, and $\int_{A_{\text {out }}}\left(\rho v_{\text {axial }}\right)_{\text {act. }} v_{\text {act. }}^{2} d A / \int_{A_{\text {out }}}\left(\rho v_{\text {axial }}\right)_{\text {invis. }} v_{\text {invis. }}^{2} d A$, respectively.

\begin{tabular}{ccccccc}
\hline $\begin{array}{c}\text { Case } \\
\text { no. }\end{array}$ & $\begin{array}{c}\dot{\boldsymbol{m}}_{\text {invis. }} \\
\left(\mathbf{k g} / \mathbf{s} \times \mathbf{1 0}^{\mathbf{8}}\right)\end{array}$ & $\begin{array}{c}\dot{\boldsymbol{m}} \\
\left(\mathbf{k g} / \mathbf{s} \times \mathbf{1 0}^{\mathbf{8}}\right)\end{array}$ & $\begin{array}{c}\text { Kinetic } \\
\text { energy } \\
\left(\mathbf{J} / \mathbf{s} \times \mathbf{1 0}^{\mathbf{4}}\right)\end{array}$ & $\begin{array}{c}\text { Inviscid } \\
\text { kinetic } \\
\text { energy } \\
\left(\boldsymbol{J} / \mathbf{s} \times \mathbf{1 0}^{\mathbf{4}}\right)\end{array}$ & $\begin{array}{c}\text { Velocity } \\
\text { efficiency } \\
(\boldsymbol{\%})\end{array}$ & $\begin{array}{c}\text { Kinetic } \\
\text { energy } \\
\text { efficiency } \\
(\boldsymbol{\%})\end{array}$ \\
\hline 2 & 1.24 & 0.75 & 2.83 & 30.7 & 60.48 & 9.22 \\
3 & 2.068 & 1.37 & 6.46 & 48.7 & 66.25 & 13.26 \\
4 & 6.15 & 4.63 & 24 & 120 & 75.28 & 20.00 \\
5 & 8.67 & 6.38 & 42 & 163.1 & 73.59 & 25.75 \\
\hline
\end{tabular}


Table 4. Effects of outlet diameter on microthruster performance. Note that $I_{s p}$ is given by Eq. (10) with $g=9.81 \mathrm{~m} / \mathrm{s}^{2}$.

\begin{tabular}{cccccc}
\hline $\begin{array}{c}\text { Case } \\
\text { no. }\end{array}$ & $\begin{array}{c}\boldsymbol{L}_{\text {out }} \\
(\mu \mathbf{m})\end{array}$ & $\boldsymbol{\theta}_{\mathbf{2}}$ & $\begin{array}{c}\boldsymbol{F}_{\boldsymbol{t}} \\
\left(\boldsymbol{N} \times \mathbf{1 0}^{\mathbf{6}}\right)\end{array}$ & $\begin{array}{c}\dot{\boldsymbol{m}} \\
\left(\mathbf{k g} / \mathbf{s} \times \mathbf{1 0}^{\mathbf{8}}\right)\end{array}$ & $\begin{array}{c}\boldsymbol{I}_{\boldsymbol{s p}} \\
(\mathbf{s})\end{array}$ \\
\hline 1 & 30 & 0 & 1.45 & 0.5 & 29.6 \\
2 & 72.2 & 8 & 2.09 & 1.0 & 21.3 \\
3 & 107.2 & 15 & 1.35 & 1.08 & 12.7 \\
4 & 119.5 & 17.5 & 1.18 & 1.07 & 11.2 \\
5 & 131.8 & 20 & 1.03 & 1.11 & 9.48 \\
6 & 156.7 & 25 & 0.81 & 1.11 & 7.45 \\
7 & 182.4 & 30 & 0.67 & 1.11 & 6.15 \\
8 & 209.7 & 35 & 0.58 & 1.11 & 5.33 \\
9 & 273.3 & 44 & 0.49 & 1.10 & 4.52 \\
\hline
\end{tabular}

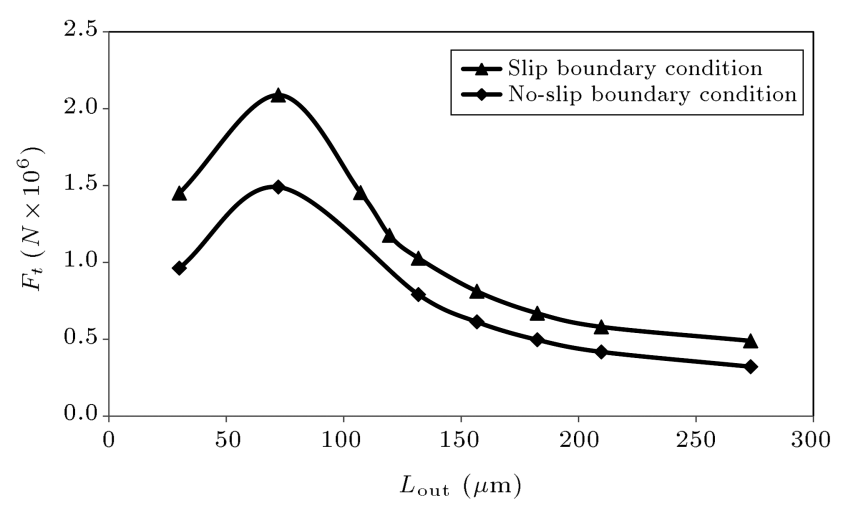

Figure 14. Effect of micronozzle outlet diameter on thrust force.

divided by the exit velocity when the flow is inviscid) is about $60 \%$ and increases with the increment of the throat diameter. The kinetic energy efficiency (actual kinetic energy of the fluid leaving the nozzle divided by the kinetic energy with isentropic expansion) is much less than the velocity efficiency and is about $9 \%$ and $26 \%$ for Cases 2 and 5, respectively.

\subsubsection{Effect of outlet diameter on micronozzle performance}

In this part of the paper, the effect of the nozzle outlet diameter on the thrust force, specific impulse, and mass flow rate passing through the micronozzle is discussed. The outlet diameter and divergence half angle are specified in Table 4. The other geometrical parameters are the same as those provided in Table 1 . The mass flow rate, thrust, and specific impulse corresponding to different outlet diameters are also provided in Table 4. They are also shown graphically in Figures 14-16.

When the divergence half angle is $8^{\circ}\left(L_{\text {out }}=\right.$ $72 \mu \mathrm{m})$, the thrust force reaches the maximum value. For outlet diameters smaller than $72 \mu \mathrm{m}$, a larger outlet diameter results in a higher mass flow rate. Therefore, the momentum and the thrust force at the exit plane

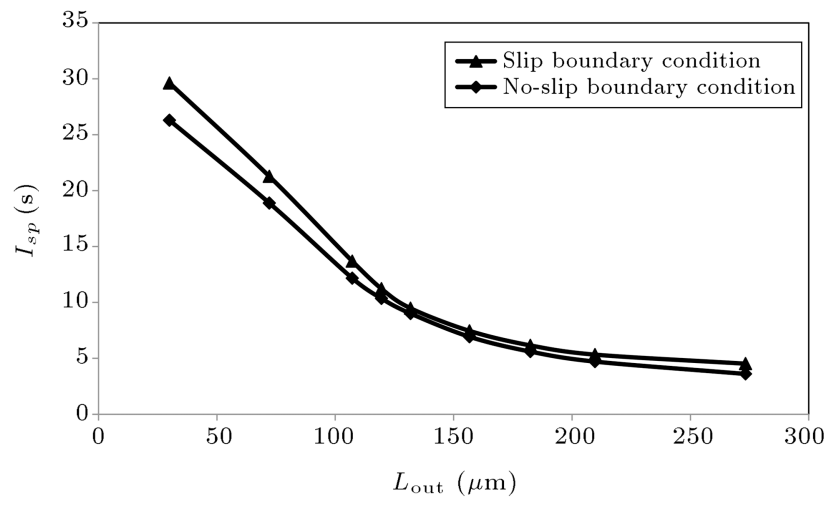

Figure 15. Effect of micronozzle outlet diameter on specific impulse. Note that $I_{s p}$ is given by Eq. (10) with $g=9.81 \mathrm{~m} / \mathrm{s}^{2}$.

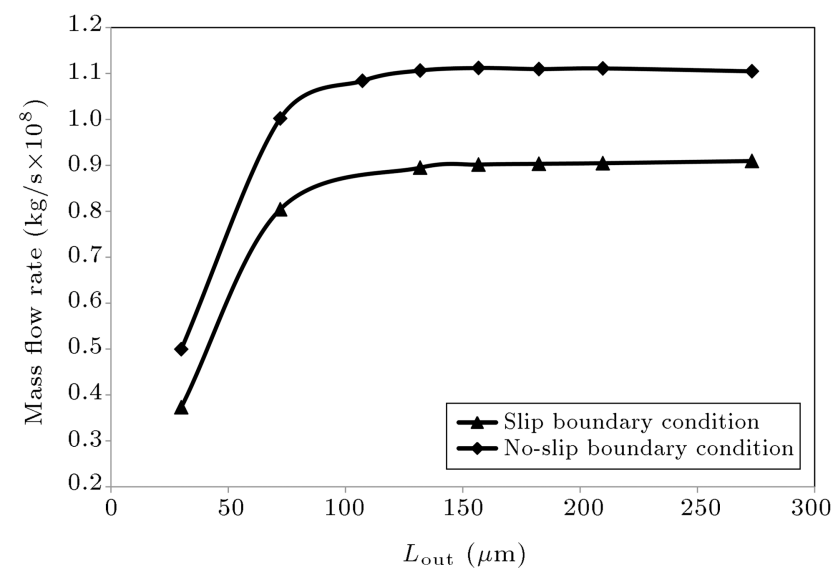

Figure 16. Effect of micronozzle outlet diameter on mass flow rate through the nozzle.

increase. However, increasing the outlet diameter beyond this value may cause flow separation in the diverging part of the micronozzle, thereby decreasing the thrust force.

Figure 16 shows that increasing the nozzle outlet diameter up to $100 \mu \mathrm{m}$ significantly increases the mass flow rate, but for values greater than $100 \mu \mathrm{m}$, the outlet diameter has nearly no effect on the mass flow rate which is a sign of the probable choking of the flow.

\section{Conclusions}

A numerical simulation of gas flow in an axisymmetric micronozzle was conducted by considering first-order slip boundary conditions. The results showed that both thrust force and specific impulse increase with the throat diameter, although specific impulse is nearly constant in the range of 30-60 $\mu \mathrm{m}$ for throat diameter. It was observed that the mass flow rate is also an increasing function of the throat diameter. In these conditions, the variations of the Mach number at the axis are changed by increasing the throat diameter from an increasing-decreasing behavior to an always 
increasing behavior. In addition, by increasing the outlet diameter, the thrust reaches its maximum at the divergence half angle of $8^{\circ}$ and is then reduced. The specific impulse in this case is shown to be a decreasing function of the outlet diameter, whereas the opposite is true for the mass flow rate. Last but not least, although the amounts of the mass flow rate, thrust force, and specific impulse are increased in the presence of rarefaction, no fundamental difference is observed between the slip and no-slip results.

\section{References}

1. Barber, R.W. and Emerson, D.R. "Challenges in modeling gas-phase flow in microchannels: From slip to transition", Heat Transfer Engineering, 27(4), pp. 3-12 (2006).

2. Harley, J.C., Huang, Y., Bau, H.H. and Zemel, J.N. "Gas flow in micro-channels", Journal of Fluid Mechanics, 284, pp. 257-274 (1995).

3. Araki, T., Kim, M.S., Iwai, H. and Suzuki, K. "An experimental investigation of gaseous flow characteristics in microchannels", Microscale Thermophysical Engineering, 6(2), pp. 117-130 (2002).

4. Arkilic, E.B., Schmidt, M.A. and Breuer, K.S. "Gaseous slip flow in long microchannels", Journal of Microelectromechanical Systems, 6(2), pp. 167-178 (1997).

5. Arkilic, E.B., Breuer, K.S. and Schmidt, M.A. "Mass flow and tangential moment um accomodation in silicon micromachined channels", Journal of Fluid Mechanics, 437, pp. 29-43 (2001).

6. Liu, J., Tai, Y.-C. and Ho, C.-M., "MEMS for pressure distribution studies of gaseous flows in microchannels", In Proceedings of the IEEE Micro Electro Mechanical Systems, pp. 209-215 (1995).

7. Choi, S.B., Barron, R.F. and Warrington, R.O., Fluid Flow and Heat Transfer in Microtubes, ASME (1991).

8. Taheri, P., Torrilhon, M. and Struchtrup, H. "Couette and poiseuille microflows: Analytical solutions for regularized 13-moment equations", Physics of Fluids, 21(1), pp. 017102 (2009).

9. Kandlikar, S.G., Garimella, S., Li, D., Colin, S. and King, M.R., Heat Transfer and Fluid Flow in Minichannels and Microchannels, Elsevier, Oxford, U.K. (2006).

10. Beskok, A. and Karniadakis, G.E. "Simulation of heat and momentum transfer in complex microgeometries", Journal of Thermophysics and Heat Transfer, 8(4), pp. 647-655 (1994).

11. Hadjiconstantinou, N.G. "The limits of Navier-Stokes theory and kinetic extensions for describing small-scale gaseous hydrodynamics", Physics of Fluids, 18(11), pp. 111301 (2006).

12. Sadeghi, A., Asgarshamsi, A. and Saidi, M.H. "Thermodynamic analysis of slip flow forced convection through a microannulus", Journal of Thermophysics and Heat Transfer, 24(4), pp. 785-795 (2010).

13. Sadeghi, A. and Saidi, M.H. "Second law analysis of slip flow forced convection through a parallel plate microchannel", Nanoscale and Microscale Thermophysical Engineering, 14(4), pp. 209-228 (2010).

14. Sadeghi, M., Sadeghi, A. and Saidi, M.H. "Buoyancy effects on gaseous slip flow in a vertical rectangular microchannel", Microfluidics and Nanofluidics, 16(12), pp. 207-224 (2014).

15. Sebastião, I.B. and Santos, W.F.N. "Numerical simulation of heat transfer and pressure distributions in micronozzles with surface discontinuities on the divergent contour", Computers and Fluids, 92, pp. 125-137 (2014).

16. Cheah, K.H. and Low, K.S. "Fabrication and performance evaluation of a high temperature co-fired ceramic vaporizing liquid microthruster", Journal of Micromechanics and Microengineering, 25(1), 015013 (2014).

17. Shen, Q., Yuan, W., Xie, J. and Chang, H. "A quantitative optimisation model for a horizontal MEMS solid propellant thruster with experimental verification", Microsystem Technologies, 22(4), pp. 847-859 (2015).

18. Louisos, W.F. and Hitt, D.L. "Numerical studies of supersonic flow in bell-shaped micronozzles", Journal of Spacecraft and Rockets, 51(2), pp. 491-500 (2014).

19. Alexeenko, A.A., Fedosov, D.A., Gimelshein, S.F., Levin, D.A. and Collins, R.J. "Transient heat transfer and gas flow in a MEMS-based thruster", Journal of Microelectromechanical Systems, 15(1), pp. 181-194 (2006).

20. Ameel, T.A., Wang, X., Barron, R.F. and Warrington Jr, R.O. "Laminar forced convection in a circular tube with constant heat flux and slip flow", Microscale Thermophysical Engineering, 1(4), pp. 303-320 (1997).

21. Aydin, O. and Avci, M. "Heat and fluid flow characteristics of gases in micropipes", International Journal of Heat and Mass Transfer, 49(9-10), pp. 1723-1730 (2006).

22. Beskok, A., Karniadakis, G.E. and Trimmer, W. "Rarefaction and compressibility effects in gas microflows", Journal of Fluids Engineering, 118(3), pp. 448-456 (1996).

23. Chen, C.I., Chen, C.K. and Lin, H.J. "Analysis of unsteady flow through a microtube with wall slip and given inlet volume flow rate variations", Journal of Applied Mechanics, Transactions ASME, 75(1), pp. 0145061-0145067 (2008).

24. Dongari, N., Agrawal, A. and Agrawal, A. "Analytical solution of gaseous slip flow in long microchannels", International Journal of Heat and Mass Transfer, 50(17-18), pp. 3411-3421 (2007).

25. Duan, Z. and Muzychka, Y.S. "Slip flow in non-circular microchannels", Microfluidics and Nanofluidics, 3(4), pp. 473-484 (2007). 
26. Duan, Z. and Muzychka, Y.S. "Compressibility effect on slip flow in non-circular microchannels", Nanoscale and Microscale Thermophysical Engineering, 11(3-4), pp. 259-272 (2007).

27. Duan, Z. and Muzychka, Y.S. "Effects of axial corrugated roughness on low Reynolds number slip flow and continuum flow in microtubes", Journal of Heat Transfer, 132(4), 041001 (2010).

28. Ghodoossi, L. and Eğrican, N. "Prediction of heat transfer characteristics in rectangular microchannels for slip flow regime and H1 boundary condition", International Journal of Thermal Sciences, 44(6), pp. 513-520 (2005).

29. Hooman, K. "A superposition approach to study slipflow forced convection in straight microchannels of uniform but arbitrary cross-section", International Journal of Heat and Mass Transfer, 51(15-16), pp. 3753-3762 (2008).

30. Jang, J. and Kim, Y.H. "Gaseous slip flow of a rectangular microchannel with non-uniform slip boundary conditions", Microfluidics and Nanofluidics, 9(2-3), pp. 513-522 (2010).

31. Jeong, H.E. and Jeong, J.T. "Extended Graetz problem including streamwise conduction and viscous dissipation in microchannel", International Journal of Heat and Mass Transfer, 49(13-14), pp. 2151-2157 (2006).

32. Khan, W.A. and Yovanovich, M.M. "Analytical modeling of fluid flow and heat transfer in microchannel/ nanochannel heat sinks", Journal of Thermophysics and Heat Transfer, 22(3), pp. 352-359 (2008).

33. Sadeghi, A., Baghani, M. and Saidi, M. "Gaseous slip flow forced convection through ordered microcylinders", Microfluidics and Nanofluidics, 15(1), pp. 73-85 (2013).

34. Sadeghi, A. and Saidi, M.H. "Viscous dissipation and rarefaction effects on laminar forced convection in microchannels", Journal of Heat Transfer, 132(7), 072401 (2010).

35. Sadeghi, A., Salarieh, H., Saidi, M.H. and Mozafari, A.A. "Effects of corrugated roughness on gaseous slip flow forced convection in microtubes", Journal of Thermophysics and Heat Transfer, 25(2), pp. 262-271 (2011).

36. Yu, S. and Ameel, T.A. "Slip flow convection in isoflux rectangular microchannels", Journal of Heat Transfer, 124(2), pp. 346-355 (2002).

37. Baghani, M. and Sadeghi, A. "Gaseous slip flow forced convection in microducts of arbitrary but constant cross section", Nanoscale and Microscale Thermophysical Engineering, 18(4), pp. 354-372 (2014).

38. Sadeghi, A., Baghani, M. and Saidi, M.H. "Gaseous slip flow mixed convection in vertical microducts withconstant axial energy input", Journal of Heat Transfer, 136(3), 032501 (2014).

39. Rossi, C., Rouhani, M.D. and Estève, D. "Prediction of the performance of a Si-micromachined microthruster by computing the subsonic gas flow inside the thruster", Sensors and Actuators, A: Physical, 87(1-2), pp. 96-104 (2000).

40. Alexeenko, A.A., Levin, D.A., Gimelshein, S.F., Collins, R.J. and Reed, B.D. "Numerical modeling of axisymmetric and three-dimensional flows in microelectromechanical systems nozzles", AIAA Journal, 40(5), pp. 897-904 (2002).

41. Alexeenko, A.A., Levin, D.A., Fedosov, D.A., Gimelshein, S.F. and Collins, R.J. "Performance analysis of microthrusters based on coupled thermal-fluid modeling and simulation", Journal of Propulsion and Power, 21(1), pp. 95-101 (2005).

42. Liu, M., Zhang, X., Zhang, G. and Chen, Y. "Study on micronozzle flow and propulsion performance using DSMC and continuum methods", Acta Mechanica Sinica, 22, pp. 409-416 (2006).

43. Chong, X. "Characteristics of micronozzle gas flows", Physics of Fluids, 19(3), 037102 (2007).

44. La Torre, F., Kenjereš, S., Moerel, J.L. and Kleijn, C.R. "Hybrid simulations of rarefied supersonic gas flows in micro-nozzles", Computers and Fluids, 49(1), pp. 312-322 (2011).

45. Watvisave, D.S., Bhandarkar, U.V. and Puranik, B.P. "Investigation of wall effects on flow characteristics of a high Knudsen number nozzle", Nanoscale and Microscale Thermophysical Engineering, 17(2), pp. 124-140 (2013).

46. Sebastião, I.B. and Santos, W.F.N. "Impact of surface discontinuities on flowfield structure of a micronozzle array", Nanoscale and Microscale Thermophysical Engineering, 18(1), pp. 54-79 (2014).

47. Kundu, P., Bhattacharyya, T.K. and Das, S. "Design, fabrication and performance evaluation of a vaporizing liquid microthruster", Journal of Micromechanics and Microengineering, 22(2), 025016 (2012).

48. Karniadakis, G., Beskok, A. and Aluru, N., Microflows and Nanoflows, Fundamentals and Simulation, Springer, New York (2005).

49. Jamison, A. and Ketsdever, A., Low Reynolds Number Performance Comparison of an Under Expanded Orifice and a DeLaval Nozzle, American Institute of Physics, Melville, NY, pp. 557-564 (2003).

50. Hirsch, C., Numerical Computation of Internal and External Flows, John Wiley \& Sons, Chichester (1990).

51. George, P.S. and Oscar, B., Rocket Propulsion Elements, 8th Ed., John Wiley \& Sons, Hoboken, NJ (2010).

52. Patankar, S. "Numerical heat transfer and fluid flow", Hemisphere Publication Company, New York (1980).

53. Bruccoleri, A.R., Leiter, R., Drela, M. and Lozano, P. "Experimental effects of nozzle geometry on flow efficiency at low Reynolds numbers", Journal of Propulsion and Power, 28(1), pp. 96-105 (2012). 


\section{Biography}

Mohammad Mahdy Heydari is a Professor of Mechanical Engineering at Malek Ashtar University of Technology, Tehran, Iran. He received his BSc degree from Khajeh Nasiredin University of Technology,
Tehran, Iran, in 1994; his MSc degree from Tarbiat Modares University, Tehran, Iran, in 1996; and his PhD degree from Sharif University of Technology, Tehran, Iran, in 2007, all in Mechanical Engineering. His research interests involve propulsion, combustion, heat transfer, and renewable energy. 